\title{
Papel del personal de salud en el diagnóstico tardío de la tuberculosis pulmonar en adultos de Medellín, Colombia
}

\author{
Marta Beatriz Gaviria, ${ }^{1}$ Hanna Marisol Henao, ${ }^{2}$ Teresita Martínez ${ }^{2}$ \\ y Elisa Bernal²
}

Forma de citar Gaviria MB, Henao HM, Martínez T, Bernal E. Papel del personal de salud en el diagnóstico tardío de la tuberculosis pulmonar en adultos de Medellín, Colombia. Rev Panam Salud Publica. 2010;27(2):83-92.

RESUMEN Objetivo. Describir el papel que desempeña el personal de salud (PS) en el retraso del diagnóstico de la tuberculosis pulmonar (TBP) en la población adulta de una ciudad colombiana. Métodos. Estudio de caso sobre el diagnóstico de la TBP en adultos de la ciudad de Medellin, Colombia, en 2007. Se recogieron datos cuantitativos y cualitativos mediante entrevistas en profundidad a 19 pacientes (11 con diagnóstico oportuno y 8 con diagnóstico tardío), la revisión de sus historias clinicas y encuestas sobre los conocimientos y las prácticas relacionadas con la TBP a 89 médicos y enfermeros de 12 instituciones prestadoras de servicios de salud. Se realizó un taller investigativo con los pacientes a fin de socializar los resultados y validar las categorías emergentes.

Resultados. Se observó un escaso conocimiento sobre la TBP y una imagen estereotipada de los pacientes que llevan a malinterpretar los sintomas y emplear tratamientos sintomáticos. Los insuficientes conocimientos del PS sobre los factores de riesgo y el cuadro clínico dificultaron el diagnóstico cuando el paciente no reflejaba el estereotipo o presentaba una enfermedad ya conocida. La desinformación sobre la transmisión de la TBP y la prescripción incorrecta de medidas de aislamiento propiciaron prácticas discriminatorias y aislamiento social y reforzaron los estereotipos y la estigmatización, lo que también limitó la búsqueda de ayuda médica. Conclusiones. El PS desempeñó un papel fundamental en el diagnóstico tardío de la TBP en los adultos estudiados. Las creencias asociadas con el estigma social y el insuficiente conocimiento sobre la enfermedad - compartidos por el PS y los pacientes-contribuyen a la construcción social del estereotipo de la enfermedad y el enfermo y reducen las posibilidades de llegar a un diagnóstico oportuno de la TBP.

Palabras clave

Tuberculosis pulmonar; diagnóstico tardío; personal de salud; estereotipo; Colombia.

En Medellín, Colombia, se realiza desde el año 2005 el seguimiento clínico, epidemiológico y serológico de una co-

\footnotetext{
Facultad Nacional de Salud Pública, Universidad de Antioquia, Medellín, Colombia. La correspondencia se debe dirigir a Marta Beatriz Gaviria, Facultad Nacional de Salud Pública, Universidad de Antioquia, calle 62 No. 52-59, piso 2, Medellín, Colombia. Correo electrónico: mgaviria@guajiros. udea.edu.co

2 Facultad de Medicina, Universidad de Antioquia, Medellín, Colombia.
}

horte de convivientes de pacientes con diagnóstico de tuberculosis pulmonar (TBP) contagiosa - llamada bacilífera-, como parte del programa estatal de control de la tuberculosis (TB) en esa ciudad (1). Según datos no publicados, en la mayoría de los casos índices del estudio se constató que hubo retraso en el diagnóstico, con una mediana del tiempo transcurrido desde el inicio de los síntomas hasta el diagnóstico de la enfermedad de
69 días, independientemente de que el cuadro clínico indicara claramente la posibilidad de TBP (L. F. García, Grupo de Inmunología Celular e Inmunogenética, Facultad de Medicina, Universidad de Antioquia, Medellín, Colombia; comunicación personal, 2006).

En un estudio realizado en Colombia en 2004 para evaluar los efectos de la reforma del sistema de salud sobre el control de la TB, se encontró un frecuente re- 
traso en el diagnóstico, lo que provocó una menor captación de pacientes con síntomas respiratorios y un mayor número de casos con cuadros clínicos avanzados al comienzo del tratamiento (2). En 2006, la incidencia de TBP en Colombia fue de 24,6 por 100000 habitantes (3), mientras que en la ciudad de Medellín ese indicador se elevaba a 32,5 por 100000 habitantes, por lo que según la Organización Mundial de la Salud (OMS) se debe considerar como una zona de alto riesgo (4).

Una de las estrategias epidemiológicas más efectivas para el control de la TB es la identificación temprana de las personas con síntomas respiratorios persistentes, independientemente del motivo de su consulta al médico (5). Se estima que entre $4 \%$ y $10 \%$ de los adultos que consultan los servicios ambulatorios de salud en los países en desarrollo con alta prevalencia de TB puede presentar tos persistente por más de 2-3 semanas; la TBP sería uno de los diagnósticos diferenciales más frecuentes en esos casos. El retraso en el diagnóstico de la TB puede elevar la letalidad, la morbilidad y la frecuencia de secuelas derivadas de la TB crónica; además, puede incrementar la incidencia debido a la mayor probabilidad de transmisión de la infección entre los contactos del paciente (6). El tiempo máximo aceptable para el diagnóstico de la TBP es de tres semanas (7).
En el tiempo de diagnóstico inciden dos factores fundamentales. El primero está asociado con el paciente y corresponde al intervalo de tiempo transcurrido entre el inicio de los síntomas y la consulta a un servicio de salud; mientras, el segundo está relacionado con el sistema de atención sanitaria y es el tiempo transcurrido entre la fecha de la consulta al servicio de salud y el inicio del tratamiento antituberculoso (8).

El significado atribuido por la población a la enfermedad y la forma en que esta se manifiesta en una cultura dada influyen notablemente en el proceso de la enfermedad, la búsqueda de atención y la curación $(9,10)$. Sin embargo, se ha comprobado que tanto en países de baja como de alta prevalencia de TB, el PS es un factor de retraso diagnóstico más importante que el paciente (11). En un estudio reciente de la Organización Panamericana de la Salud (OPS) y la OMS, en el que participaron varias escuelas de medicina de América Latina, se comprobó que la enseñanza sobre la TB es insuficiente y que con frecuencia los conocimientos brindados son limitados, fragmentados y desactualizados (12). Se constató, además, que la baja motivación del PS debido a las inadecuadas condiciones laborales y los escasos programas de educación continuada influían negativamente en el diagnóstico y el tratamiento de la TBP (12).
El retraso diagnóstico de la TBP es un asunto complejo que abarca dimensiones históricas, sociales, culturales, económicas, afectivas y ecológicas de los pacientes, así como algunas características del sistema de salud y la relación pacientePS, todas estas tejidas en intrincadas relaciones. El análisis en profundidad de un caso y su contexto puede contribuir a identificar los componentes, las relaciones y la forma en que interactúan los diversos factores que intervienen en este complicado proceso (13).

En este estudio se analiza y describe el papel que desempeña el PS en el retraso del diagnóstico de la TBP en la población adulta de una ciudad colombiana.

\section{MATERIALES Y MÉTODOS}

Se realizó un estudio de caso sobre el proceso de diagnóstico de la TBP en adultos de la ciudad de Medellín, Colombia, en 2007. Para ello se configuraron cuatro subunidades de análisis: el enfermo, la enfermedad, la atención previa y el diagnóstico final, sobre los que se recolectaron datos cuantitativos y cualitativos. Las fuentes de evidencia y los indicadores empleados para cada subunidad de análisis se muestran en el cuadro 1.

Los participantes se seleccionaron de los 438 pacientes con TBP diagnosticados entre 2005 y 2007 en instituciones presta-

\section{CUADRO 1. Indicadores y fuentes de evidencia para cada subunidad de análisis, Medellín, Colombia, 2007}

\begin{tabular}{|c|c|c|}
\hline $\begin{array}{l}\text { Subunidad } \\
\text { de análisis }\end{array}$ & Indicadores & Fuente \\
\hline El enfermo & $\begin{array}{l}\text { - Conocimientos del paciente sobre la enfermedad previos al diagnóstico } \\
\text { - Estereotipos construidos por el paciente (categoría emergente) } \\
\text { - Percepción del paciente de estigmatización y discriminación (categoría emergente) } \\
\text { - Autoestigmatización } \\
\text { - Estigmatización y discriminación por familiares y otras personas cercanas } \\
\text { - Estigmatización y discriminación por el personal de salud } \\
\text { - Recomendaciones sobre el proceso de diagnóstico desde su experiencia como paciente (categoría } \\
\text { emergente) }\end{array}$ & $\begin{array}{l}\text { Entrevistas a pacientes } \\
\text { Taller con pacientes }\end{array}$ \\
\hline La enfermedad & $\begin{array}{l}\text { - Interpretación del paciente de los síntomas y signos } \\
\text { - Conocimientos del personal de salud sobre los factores de riesgo y el cuadro clínico } \\
\text { - Estereotipos construidos por el personal de salud (categoría emergente) } \\
\text { - Conocimiento del personal de salud sobre las medidas de aislamiento }\end{array}$ & $\begin{array}{l}\text { Entrevistas a pacientes } \\
\text { Taller con pacientes } \\
\text { Encuestas al personal de salud }\end{array}$ \\
\hline La atención previa & $\begin{array}{l}\text { - Comportamiento de búsqueda de ayuda por parte del paciente } \\
\text { - Razones del paciente para consultar o no al médico } \\
\text { - Tratamiento sintomático sin prescripción médica }\end{array}$ & $\begin{array}{l}\text { Historias clínicas de los pacientes } \\
\text { Entrevistas a pacientes } \\
\text { Taller con pacientes }\end{array}$ \\
\hline Diagnóstico final & $\begin{array}{l}\text { - Conocimientos del personal de salud acerca del proceso de diagnóstico } \\
\text { - Atención sanitaria desde la primera consulta y el diagnóstico } \\
\text { - Exámenes de laboratorio y radiología realizados para establecer el diagnóstico } \\
\text { - Demora atribuible a los servicios de salud } \\
\text { - Tratamiento sintomático con prescripción médica } \\
\text { - Percepción del paciente sobre la oportunidad diagnóstica }\end{array}$ & $\begin{array}{l}\text { Encuestas al personal de salud } \\
\text { Historias clínicas de los pacientes } \\
\text { Entrevistas a pacientes } \\
\text { Taller con pacientes }\end{array}$ \\
\hline
\end{tabular}


doras de servicios de salud (IPS), según los registros de la base de datos de un estudio de cohorte previo (1). Para lograr la mayor variabilidad posible y abarcar diversas perspectivas del problema se empleó una estrategia de muestreo por propósito $(14,15)$, por lo que se tomaron los pacientes e IPS con valores de oportunidad diagnóstica extremos. Los pacientes se clasificaron en "tardíos" - los que recibieron el diagnóstico después de 44 días de iniciados los síntomas respiratorios, es decir, después de un mes de lo recomendado- y "oportunos", los que recibieron el diagnóstico a los 44 días o menos. Este criterio de un mes como tiempo máximo aceptable de demora para el diagnóstico se tomó por consenso de los autores, ya que no hay una recomendación establecida en la literatura científica. Un criterio adicional de variabilidad fue que los participantes tuvieran características sociodemográficas diversas.

Se seleccionaron 19 pacientes (11 con diagnóstico oportuno y 8 con diagnóstico tardío) con los siguientes criterios de inclusión: casos confirmados por técnicas microbiológicas, de ingreso reciente a la cohorte (para disminuir el sesgo de memoria), residentes en Medellín al momento del estudio y que aceptaran participar voluntariamente (el porcentaje de respuesta fue de $100 \%$ ). Las características sociodemográficas de los pacientes se describen en el cuadro 2.

Igualmente, se seleccionaron 14 IPS (siete con la mayor proporción de pacientes con diagnóstico oportuno y siete con la mayor proporción de pacientes con diagnóstico tardío) con los siguientes criterios de inclusión: pertenecer al primer nivel de atención de salud, estar localizadas en Medellín y que aceptaran participar; 12 IPS aceptaron participar (seis de cada subgrupo), para un porcentaje de respuesta de $85,7 \%$. Se invitó a participar a todo el personal de salud que laboraba en las IPS. Al final aceptaron participar 89 trabajadores: 48 médicos generales y 41 enfermeros (cuadro 3 ).

Las entrevistas en profundidad a los pacientes - según una guía semiestructurada elaborada por el equipo de investigación - se realizaron en sesiones de 90 a 120 minutos en sus domicilios y estuvieron a cargo de dos de las investigadoras. El cuestionario contenía preguntas sobre el paciente (situación socioeconómica, percepción de su estado de salud, contactos previos con el sistema de salud, infor-

CUADRO 2. Características sociodemográficas de los pacientes participantes en el estudio, Medellín, Colombia, 2007

\begin{tabular}{|c|c|c|c|c|c|c|}
\hline \multirow[b]{2}{*}{ Característica } & \multicolumn{2}{|c|}{$\begin{array}{c}\text { Total } \\
(n=19)\end{array}$} & \multicolumn{2}{|c|}{$\begin{array}{l}\text { Diagnóstico oportuno } \\
\qquad(n=11)\end{array}$} & \multicolumn{2}{|c|}{$\begin{array}{c}\text { Diagnóstico tardío } \\
\quad(n=8)\end{array}$} \\
\hline & No. & $\%$ & No. & $\%$ & No. & $\%$ \\
\hline \multicolumn{7}{|l|}{ Sexo } \\
\hline Hombres & 10 & 52,6 & 7 & 63,6 & 3 & 37,5 \\
\hline Mujeres & 9 & 47,4 & 4 & 36,4 & 5 & 62,5 \\
\hline \multicolumn{7}{|l|}{ Edad (años) } \\
\hline$<35$ & 10 & 52,6 & 6 & 54,5 & 4 & 50,0 \\
\hline$\geq 35$ & 9 & 47,4 & 5 & 45,5 & 4 & 50,0 \\
\hline \multicolumn{7}{|l|}{ Seguro de salud } \\
\hline Régimen contributivo & 11 & 57,9 & 7 & 63,6 & 4 & 50,0 \\
\hline Régimen subsidiado & 3 & 15,8 & 0 & 0,0 & 3 & 37,5 \\
\hline No asegurado & 5 & 26,3 & 4 & 36,4 & 1 & 12,5 \\
\hline \multicolumn{7}{|l|}{ Tipo de trabajo } \\
\hline Remunerado ${ }^{a}$ & 10 & 52,6 & 7 & 63,6 & 3 & 37,5 \\
\hline No remunerado & 9 & 47,4 & 4 & 36,4 & 5 & 62,5 \\
\hline
\end{tabular}

Fuente: entrevistas a los pacientes.

a Profesor, enfermera, obrero, vendedor informal, taxista o empleada doméstica.

b Estudiante, ama de casa, jubilado, pensionado por discapacidad o desempleado.

CUADRO 3. Características sociodemográficas del personal de salud participante en la investigación, Medellín, Colombia, 2007

\begin{tabular}{|c|c|c|c|c|c|c|}
\hline \multirow[b]{2}{*}{ Característica } & \multicolumn{2}{|c|}{$\begin{array}{c}\text { Total } \\
(n=89)\end{array}$} & \multicolumn{2}{|c|}{$\begin{array}{l}\text { Médicos } \\
(n=48)\end{array}$} & \multicolumn{2}{|c|}{$\begin{array}{c}\text { Personal de } \\
\text { enfermería } \\
\quad(n=41)\end{array}$} \\
\hline & No. & $\%$ & No. & $\%$ & No. & $\%$ \\
\hline \multicolumn{7}{|l|}{ Sexo } \\
\hline Hombres & 25 & 28,1 & 25 & 52,1 & 0 & 0,0 \\
\hline Mujeres & 64 & 71,9 & 23 & 47,9 & 41 & 100,0 \\
\hline \multicolumn{7}{|l|}{ Edad (años) } \\
\hline$<35$ & 43 & 48,3 & 26 & 54,2 & 17 & 41,5 \\
\hline$\geq 35$ & 46 & 51,7 & 22 & 45,8 & 24 & 58,5 \\
\hline \multicolumn{7}{|c|}{ Tipo de institución sanitaria } \\
\hline Pública & 52 & 58,4 & 25 & 52,1 & 27 & 65,8 \\
\hline Privada & 37 & 41,6 & 23 & 47,9 & 14 & 34,2 \\
\hline \multicolumn{7}{|c|}{ Experiencia laboral (años) } \\
\hline$<10$ & 53 & 59,6 & 32 & 66,7 & 21 & 51,2 \\
\hline$\geq 10$ & 36 & 40,4 & 16 & 33,3 & 20 & 48,8 \\
\hline \multicolumn{7}{|l|}{ Tipo de contrato } \\
\hline Estable $^{a}$ & 48 & 53,9 & 23 & 47,9 & 25 & 61,0 \\
\hline No estableb & 41 & 46,1 & 25 & 52,1 & 16 & 39,0 \\
\hline
\end{tabular}

Fuente: encuestas al personal de salud.

a Contrato por término indefinido.

${ }^{\mathrm{b}}$ Otro tipo de contratación: temporal, prestación de servicios o cooperativas de profesionales.

mación previa y actual sobre la TBP y sentimientos sobre la enfermedad), la enfermedad (primeros síntomas, cuadro clínico y su interpretación), la atención recibida (búsqueda de ayuda, red de apoyo, percepción del trato del PS, diagnósticos y tratamientos previos, y percepción del acceso a los servicios de salud adecuados) y el diagnóstico final (cómo se llegó a él, cómo y quién se lo informó y cómo se percibió la oportunidad).

Adicionalmente, dos auxiliares de investigación revisaron las historias clíni- cas en las instituciones identificadas por el paciente en la entrevista y se registraron los datos de cada uno de los cuidados recibidos (médicos y de enfermería) en los servicios ambulatorios y hospitalarios consignados, entre el inicio de los síntomas y el momento del diagnóstico. Se recabó información sobre el enfermo (identificación, características sociodemográficas, antecedentes personales y familiares), la enfermedad (anamnesis, exámen físico, pruebas de laboratorio y radiología), la atención recibida (lugar y 
fecha de atención, tipo de profesional, servicio e impresiones diagnósticas), conducta médica (prescripción de medicamentos, exámenes de laboratorio y radiología, y hospitalización) y de enfermería (recomendaciones para el paciente y convivientes) y el diagnóstico final recibido (lugar y fecha de atención, tipo de profesional, servicio, exámenes de laboratorio y radiología y fecha de diagnóstico). Por último, al final del estudio los pacientes participaron en un taller investigativo con el propósito de socializar los resultados y validar las categorías emergentes (15).

El PS participante respondió una encuesta sobre sus conocimientos y prácticas relacionados con el diagnóstico y el tratamiento de infecciones respiratorias en adultos. Con el propósito de reducir posibles sesgos de información, no se hizo explícito que se indagaría específicamente sobre la TBP. El formulario, elaborado por el equipo de investigación con la ayuda de expertos, tuvo como referencia las guías nacionales de atención de la TBP (16). Esta encuesta se respondió de forma autoadministrada, anónima y confidencial en las instalaciones de las IPS en aproximadamente $45 \mathrm{minu}-$ tos. La encuesta constaba de dos partes. En la primera se presentaron casos clínicos de infección respiratoria en adultos - los enunciados se configuraron a partir de los datos de las entrevistas y las historias clínicas de los pacientes participantes considerados como los más ilustrativos de retraso diagnóstico- y se preguntó sobre el enfoque inicial, la impresión diagnóstica y la conducta a seguir. En la segunda se indagó acerca de los conocimientos y las prácticas sobre TBP, en particular, la presentación clínica, los factores de riesgo, el diagnóstico y las medidas de aislamiento.

Las entrevistas con los pacientes se grabaron y transcribieron antes de procesarlas mediante el sistema Atlas Ti versión 3.2.1. Los datos se codificaron en categorías y subcategorías teóricas (incluidas en la guía) y emergentes (que surgieron durante la entrevista) y se realizó una descripción inicial. Después de esto se construyeron categorías con mayores niveles de agregación. La interpretación se realizó según los patrones y las relaciones establecidas entre las categorías para determinar las subcategorías emergentes de mayor importancia. La presentación de los resultados cualitativos se hizo de forma narrativa a partir de los testimonios más ilustrativos de las diversas perspectivas de los participantes (15).

La información sobre las historias clínicas de los pacientes y la encuesta al PS se procesaron y analizaron mediante el paquete informático SPSS versión $15.0 \mathrm{y}$ se calcularon las medidas de las frecuencias y estadísticos de tendencia central y dispersión. No se aplicaron pruebas de significación a las diferencias encontradas entre los subgrupos, porque el muestreo no fue aleatorio y la intención de generalización de resultados no es de tipo estadística (17).

A partir de las distintas fuentes de evidencia (entrevistas, historias clínicas, taller con los pacientes y encuestas al PS) se realizó un análisis embebido de los aspectos específicos del caso (subunidades de análisis) y se construyó un modelo teórico con los factores y relaciones encontrados (14). Se garantizó la calidad del estudio mediante la triangulación o convergencia de las diversas fuentes de evidencia, a partir de la relación directa de los datos (13) y el chequeo o validación con los pacientes de las categorías emergentes (15).

Esta investigación recibió la aprobación previa del Comité para el Desarrollo de la Investigación de la Universidad de Antioquia.

\section{RESULTADOS}

\section{El enfermo}

Conocimientos sobre la enfermedad antes del diagnóstico. Antes del diagnóstico, los pacientes tenían escasos conocimientos sobre la enfermedad y predominaban las creencias erróneas y conceptos estereotipados que la asociaban con gravedad, muerte, pobreza y exclusión social. Una vendedora informal de 42 años diagnosticada tardíamente afirmó: "Yo de la TB no sabía nada, pues uno siempre ve las cosas como tan lejos de uno [. . .]". Un profesor de 25 años de edad que tuvo un diagnóstico oportuno pensaba que era "una enfermedad muy contagiosa, que hay que poner mucho cuidado; muchos dicen que les da a los más pobres, a los marginados, por desaseo [. . . ]". Un ama de casa de 24 años con diagnóstico tardío narró que al conocer su diagnóstico, la madre le dijo: "Yo no pensé que usted tuviera una enfermedad de esas tan grave [. . .]. Yo he escuchado mencionar que es muy peligrosa, lo puede matar a uno".
Percepción de estigmatización y discriminación. En las entrevistas, los pacientes se refirieron a la discriminación y la estigmatización que percibían de parte de familiares, otras personas cercanas e incluso del PS que les brindó atención y ellos mismos. Aislarse de sus familiares y amigos $\mathrm{u}$ ocultar su diagnóstico fueron prácticas que adoptaron los pacientes como expresión del temor a contagiar a otros, pero también a ser discriminado o rechazado. Una vendedora informal de 31 años con diagnóstico tardío compartió con mucho dolor el siguiente testimonio: "[al ser notificada del diagnóstico de TBP] automáticamente comencé a aislarme, primero por la enfermedad y segundo por lo mal que me sentía. No quería que nadie me viera, pues; que supiera que yo estaba enferma [...]. Cuando alguien está enfermo de esto, mucha gente suele aislarnos, y no quería que de pronto eso me pasara a mí". Y al ingresar a la consulta en el programa de control de TB, señaló: "El doctor me hacía sentir mal, me decía que no me le acercara, que cogiera la silla y me retirara [. . . . Un día yo le dije: 'si quiere me salgo y me hace la consulta desde afuera'. Me dijo: 'no, simplemente deje la puerta abierta' [. . .]".

Recomendaciones a partir de su experiencia en el proceso de diagnóstico. En las entrevistas surgió que la enfermedad significó, además de dolor y sufrimiento, un aprendizaje relevante sobre su cuidado y el de los demás y los pacientes adoptaron un papel activo para aportar recomendaciones a fin de evitar retrasos en el diagnóstico. Un ama de casa de 24 años que tuvo un diagnóstico tardío expresó: "Si por mi fuera, yo me haría en un tablado en toda la mitad de la calle en mi barrio y hablaría de la enfermedad, de lo duro que yo pasé. A mí no me daría pena decirlo porque de lo que uno vivió, pues, la experiencia es la que le llega a los demás. Por eso para mí es muy importante. Yo le diría a una persona que se cuide un poquito más y que luche por una forma o por otra a hacerse esos exámenes". Un taxista de 29 años diagnosticado oportunamente sugirió que las autoridades sanitarias debían brindar información sobre la TBP de forma suficiente y clara, "[. . .] alertando a las personas para que se prevengan, sepan los síntomas y aprendan que así pueden exigir un examen, que ellas también tienen derecho". Un pensionado por discapacidad, de 53 años de edad, con diagnóstico 
tardío propuso que debía mejorarse la práctica médica: "[...] ponerle un poquito más de cuidado al paciente, pues parece que trabajaran mecánicamente [...]. El mayor error que yo he encontrado en la mayoría de ellos es que pecan de sabios. Creen que como ellos estudiaron, el bruto es uno. Usted va y le dice: 'doctor, es que mire que [...]'; 'ah, no, tranquilo, que eso se quita'. Ellos nunca se preocupan por estudiar más el problema". Un profesor de 28 años con diagnóstico tardío planteó la necesidad de cambiar el modelo de atención: "Después de haber pasado por muchas consultas, creo que los médicos imponen una distancia tal que les impide conocer lo que le sucede realmente al paciente [...]. Esto ocurre en parte por los condicionamientos del sistema de salud; parece que los vuelve más ciegos. ¡Y esto debería cambiar!"

\section{La enfermedad}

Sospecha de la enfermedad por parte del paciente. Los pacientes no pensaron que tenían TBP; creyeron que se trataba de un problema de salud pasajero e interpretaron sus signos y síntomas como una infección viral común o como molestias respiratorias por cambios climáticos. Una vendedora informal de 31 años con diagnóstico tardío intuyó que era "la tos que le da a las personas por una gripa [...], no pensé que era nada malo". Al presentarse un mayor deterioro de su estado general, como pérdida marcada de peso y hemoptisis, un paciente desempleado de 53 años con diagnóstico tardío indicó que pensó en una enfermedad que podría llevarlo a la muerte: "[. . .] yo pensé que el problema era más grave [...], como yo había fumado pensé que era un cáncer".

Sospecha de la enfermedad por parte del PS. En la encuesta aplicada al PS hubo una baja proporción de respuestas correctas sobre factores de riesgo y cuadro clínico de la TBP. Por ejemplo, 88,8\% desconocía que "la pérdida de peso no es frecuente en las fases iniciales de la enfermedad"; 50,6\% dijo que "no todos los pacientes con TBP tienen tos" y 30,3\% pensaba que "los síntomas respiratorios pueden mejorar momentáneamente con antibióticos (macrólidos o betalactámicos)". Todos estos errores podrían reducir la capacidad de acercarse al diagnóstico de esta enfermedad y propiciar la construcción de una imagen clínica estereotipada de los pacientes, asociada con un cuadro clínico llamativo con deterioro del estado general. En la presentación de casos clínicos se observó que el PS tuvo una mayor sospecha diagnóstica de TBP cuando las descripciones apuntaban a una visión estereotipada del enfermo. Por ejemplo, solo $36,0 \%$ de los encuestados consideró como TBP el siguiente caso clínico: "hombre joven, trabajador y estudiante universitario, perteneciente al régimen contributivo; con rápida evolución de síntomas respiratorios y en el examen físico con crépitos en la base pulmonar izquierda", probablemente porque no "encajaba" con el estereotipo. A diferencia de otro caso presentado: "hombre de 34 años, afrodescendiente, habitante de la calle, consumidor de sustancias psicoactivas; había sido llevado al servicio de urgencias por trauma abdominal y durante la hospitalización informó tener tos persistente y adenomegalias", en el que 65,2\% de los encuestados - con una mayor proporción de médicos $(79,2 \%)$ - optaron por un diagnóstico probable de TBP, posiblemente por las condiciones de exclusión social que generaron una mayor sospecha clínica de la enfermedad por ajustarse más al estereotipo.

La baja proporción de respuestas correctas sobre los casos clínicos indica que el PS tiene un estereotipo social de la persona afectada por TBP caracterizado por la pobreza y la exclusión social. En dos pacientes que acudieron a los programas preventivos no se reconocieron las condiciones clínicas de base como factores de riesgo para el desarrollo de la TBP. Es así como solo 19,1\% del PS encuestado pensó en la TBP al analizar el caso de un "hombre de 53 años asegurado en el régimen subsidiado de salud, diabético, que acudía regularmente al control del programa preventivo, que hacía algún tiempo presentaba tos y en los dos últimos controles le encontraron cifras elevadas de glucemia a pesar de haber cumplido con el tratamiento farmacológico y nutricional; al evaluarlo tenía marcada pérdida de peso". Asimismo, 41,6\% tuvo sospecha diagnóstica de TBP en el caso de una "mujer de 24 años perteneciente al régimen subsidiado, que asistió durante la gestación al control prenatal y que después de dos semanas del parto consultó a un hospital por tos abundante desde hacía varias semanas, mal estado general y en el exa- men físico presentó hipoventilación en ambos campos pulmonares". Esta baja proporción de sospecha diagnóstica podría deberse a que el PS tiende a fijar la atención sobre el riesgo específico que es objeto de atención en un programa dado.

Conocimientos y prácticas del PS sobre las medidas preventivas. Una elevada proporción del PS tenía conocimientos erróneos sobre las medidas preventivas de asilamiento apropiadas para la TBP, pues $78,7 \%$ consideró que "compartir alimentos con una persona con TBP es un factor de riesgo para infectarse" y $68,5 \%$ dijo que "para prevenir el contagio es necesario separar la vajilla y los cubiertos de los pacientes". Asimismo, en las entrevistas, los pacientes identificaron como una práctica generalizada en el PS la exigencia de medidas obsoletas para la prevención del contagio que refuerzan la discriminación y el aislamiento social de las personas afectadas y perpetúan el estigma, como el uso de la mascarilla y la separación y desinfección de la vajilla y los cubiertos. Una empleada doméstica de 36 años diagnosticada oportunamente narró que "el médico allá empezó a decir que eso es una enfermedad muy contagiosa, que tiene que separar los pocillos, la comida y la ropa, y dormir en una habitación aparte de los niños. Todo eso me hacía sentir mal [. . . es como si a usted le dicen que tiene sida y que va a morir de eso".

\section{Atención previa}

Búsqueda de ayuda y tratamiento sintomático por parte del paciente. En las entrevistas, los pacientes relataron que antes de ir a un servicio de salud acudieron al tratamiento sintomático sin prescripción médica, uso de remedios caseros, automedicación o consulta al farmacéutico. Estas prácticas pudieron dilatar la consulta temprana a los servicios de salud. Un ama de casa de 57 años con diagnóstico tardío afirmó que había "[.. .] tomado muchas bebidas, de saúco [hierba medicinal frecuente en la región], cosas que me decían; tome miel de abejas con limón y mantequilla porque era muy bueno para la tos [. . . " y otra ama de casa de 24 años con diagnóstico tardío relató que "[. . .] tomé otro jarabe y tampoco; compramos todo lo que decían en la televisión y a mí nada me sirvió". La búsqueda de ayuda médica se vio propiciada por la persistencia de los síntomas, 
la duración y la gravedad de la discapacidad asociada y cuando el cuadro clínico era muy manifiesto y respondía al estereotipo del enfermo de TBP. Así, un pensionado de 53 años con diagnóstico tardío dijo que consultó los servicios de salud porque "¡me quedé sin un aliento! [...] me cansaba caminando, desayunando, bañándome [...]". Una enfermera de 32 años con diagnóstico oportuno dijo sobre la causa de consultar un médico: "creo que fue por el síntoma más avanzado, la hemoptisis; yo creo que si hubiera comenzado con la fiebre yo nunca hubiera pensado en la TB; por eso consulté rápido, pues nunca pensé que podía enfermarme de esto".

\section{Diagnóstico final}

Conocimientos y prácticas diagnósticas del PS. El insuficiente conocimiento sobre el diagnóstico es uno de los factores relacionados con el PS que pueden influir en el retraso del diagnóstico y la atención del paciente. A pesar de que 93,3\% del PS encuestado sabía que "si se determina que un paciente es sintomático respiratorio, el examen inicial es una prueba de baciloscopia seriada de esputo", solo $64,0 \%$ aceptó que "un sintomático respiratorio es aquel que presenta tos y/o expectoración por más de 15 días". No tener una definición clara de qué se considera un paciente con síntomas respiratorios puede llevar a una inapropiada prescripción de la prueba de baciloscopia en esputo. El conocimiento de técnicas diagnósticas complementarias también presentó dificultades, pues solamente 70,8\% del PS sabía que "una radiografía de tórax normal no descarta el diagnóstico de $\mathrm{TBP}^{\prime \prime}$ y solo 57,3\% sabía que "a un paciente sintomático respiratorio se le indica un cultivo para micobacterias en esputo si el paciente se ha realizado tres pruebas de baciloscopia seriadas de esputo sin que se detecten bacilos positivos a la tinción de ZiehlNeelsen y persiste la sospecha clínica".

Atención sanitaria desde la primera consulta y el diagnóstico. Una inadecuada impresión diagnóstica, con escasa sospecha de TBP, pudo llevar a no prescribir una prueba de baciloscopia y al tratamiento sintomático de los pacientes con cuadros respiratorios. Como se puede observar en la figura 1, la mayor parte $(68,6 \%)$ del tiempo entre el inicio de los síntomas y el diagnóstico en los pacientes con diagnóstico tardío (del 12 al 19), transcurrió después de haber consultado un servicio de salud. Por tanto, el retraso diagnóstico podría atribuirse principalmente a factores relacionados con la atención sanitaria. En este grupo de pacientes, el diagnóstico se consiguió entre 23 y 457 días después de la primera consulta. Otro elemento llamativo es que el diagnóstico de TBP no siempre se realizó de manera ambulatoria (que es lo esperado

FIGURA 1. Tiempo transcurrido entre el inicio de los síntomas y el momento del diagnóstico de tuberculosis pulmonar, antes y después de consultar a un servicio de salud, Medellín, Colombia, 2007

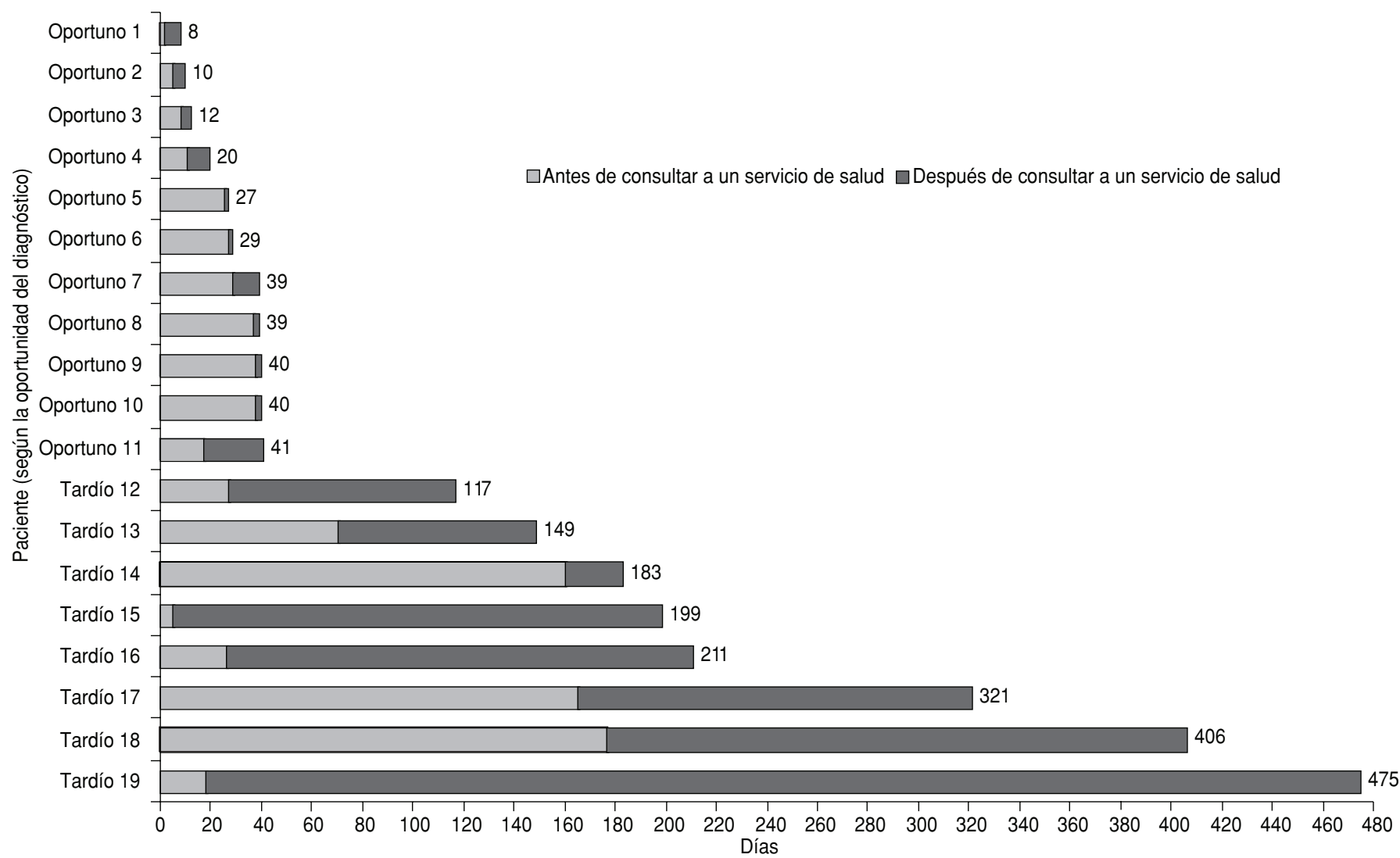


según las normas del programa): la mitad de estos pacientes recibió el diagnóstico mientras se encontraban hospitalizados. En las historias clínicas se registró que a solo siete de los 19 pacientes estudiados se les indicó realizarse una prueba de baciloscopia al llegar por primera vez a la consulta con síntomas respiratorios, a nueve pacientes se les prescribieron otras técnicas diagnósticas (radiografía de tórax, hemoleucograma y sedimentación, y endoscopia digestiva) y a los tres restantes no se les ordenó ninguna medida diagnóstica. Por último, se prescribió un tratamiento sintomático de los pacientes, principalmente con medicamentos analgésicos, antiinflamatorios, antihistamínicos, broncodilatadores, antitusivos, expectorantes y antibióticos. Una paciente de 24 años con diagnóstico tardío señaló que "la doctora me mandó inhaladores y un jarabe que era muy caro [...]. Me dijo que si hacía lo que ella decía, con eso me paraba". Asimismo, otra ama de casa de 57 años con diagnóstico tardío narró que "el médico me mandó acetaminofén y me dijo: 'Tómese bien juiciosa la droga para el azúcar y estas pastillitas'. Yo me tomé todas esas 20 pastas de antibiótico y me empeoré".

Percepción del paciente sobre la oportunidad diagnóstica. Algunos de los pacientes que acudían a controles preventivos identificaron como posible causa del retraso en su diagnóstico el hecho de estar embarazada o presentar diabetes o hipertensión arterial, ya que eso pudo haber "distraído" al médico. Uno de ellos, de 53 años, afirmó que "de pronto por estar pendiente del problema del azúcar, el médico se preocupaba mucho por el problema renal. Yo creo que esa es una de las razones por las cuales descuidó la inquietud que yo le llevaba con relación a la tos [. . .]"; y otro de 76 años explicó: "Pues, porque tal vez no me examinaban en una forma posible de encontrarme la TBP que yo tenía, pues se limitaban al control de la presión".

En la figura 2, se presenta un esquema que resume las categorías y las subcategorías identificadas como factores del diagnóstico tardío de TBP en adultos y las relaciones implicadas en ese problema. Se puede comprobar la importancia de la comprensión del problema, la percepción de estigmatización, la discriminación por parte de los pacientes y los estereotipos de la enfermedad, tanto por parte del PS como de los pacientes. Estas categorías surgieron durante el análisis de los datos cualitativos.

\section{DISCUSIÓN}

La información recolectada de los pacientes con TBP que participaron en este estudio puede reflejar los conocimientos y creencias de la población general de la ciudad, de la que forma parte. La construcción social de consensos relacionados con la salud y la enfermedad - específicamente sobre la TBP y las personas afectadas por ella- se hace a partir de comentarios, experiencias previas, antecedentes familiares y medios de comunicación, entre otros (18). Lamentablemente, la información científica se integra lentamente a esta construcción, lo que favorece las prácticas inapropiadas

FIGURA 2. Factores y relaciones implicadas en el retraso diagnóstico de la tuberculosis pulmonar (TBP), Medellín, Colombia, 2007

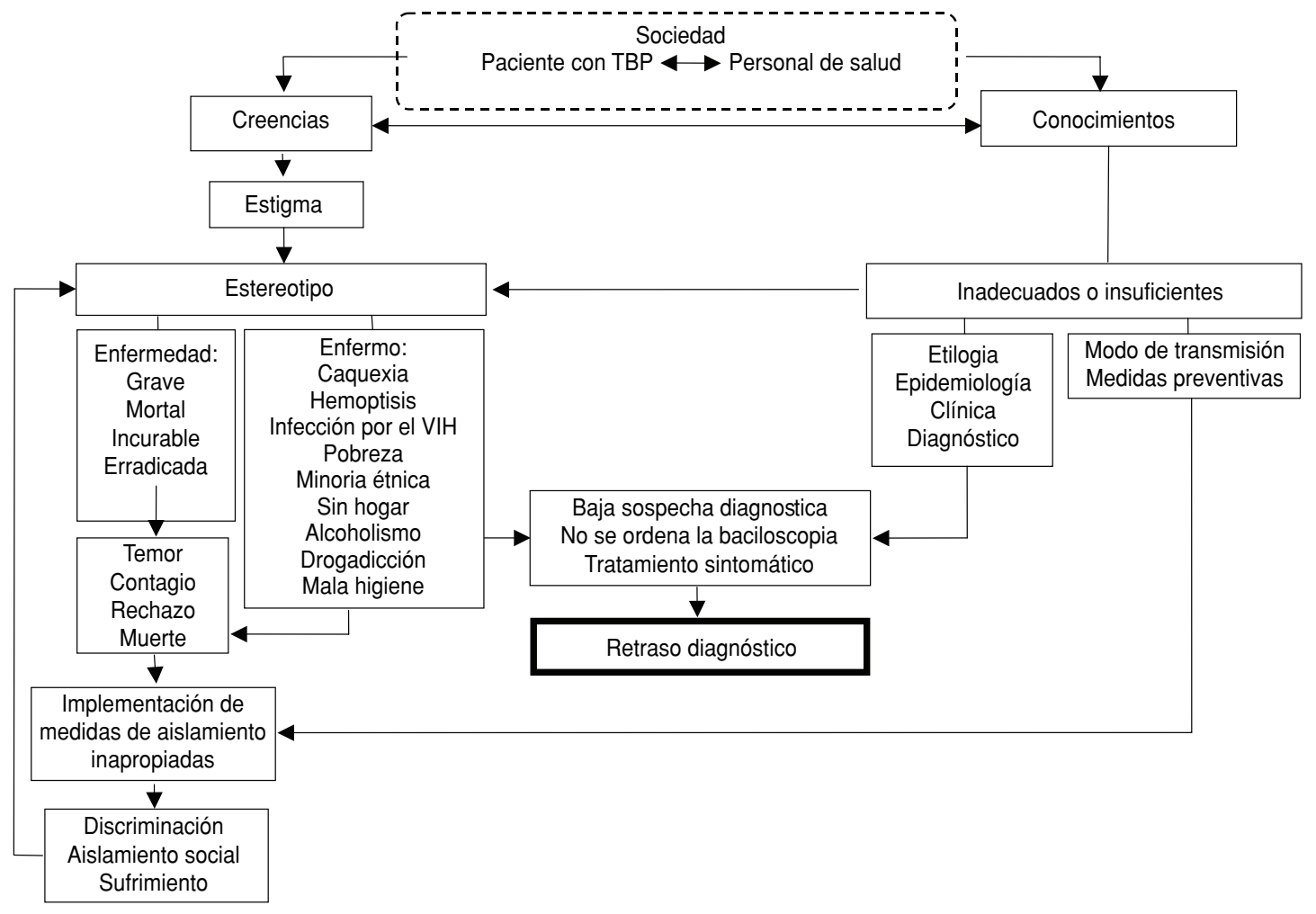


que en ocasiones resultan en el aislamiento social y el sufrimiento (19).

El insuficiente conocimiento sobre la TBP encontrada entre los pacientes de este estudio, ya observada en investigaciones anteriores (20-22), influye negativamente en el proceso de diagnóstico, de manera similar a lo que ocurre con el PS. Así, desconocer la etiología, la epidemiología, el cuadro clínico y el diagnóstico dificulta la búsqueda activa de casos con síntomas respiratorios y el inicio del tratamiento adecuado. En conjunto, esto puede conducir al retraso en el diagnóstico, lo que a su vez extiende la cadena de transmisión y lleva al aumento en la incidencia de TB. Simultáneamente, desconocer el mecanismo de transmisión y las medidas para prevenir el contagio puede conducir a prácticas higienistas exageradas y obsoletas que pueden perpetuar el aislamiento social y la discriminación.

Las expresiones de estigmatización y discriminación percibidas por los pacientes, que fueron categorías emergentes en este estudio, repercuten no solo en un mayor sufrimiento para ellos y sus familiares, sino que pueden provocar demoras en el diagnóstico. Para Goffman, el estigma es una situación que impide que la persona que lo padece tenga una plena aceptación social, justifica la inferioridad de quien lo padece, expone exageradamente el peligro que esa persona representa para los demás, enfatiza la relación entre un atributo y un estereotipo y resulta en un tratamiento injusto y discriminatorio (23). Hay pocos trabajos publicados acerca de la determinación de las causas -más que de la existencia o los efectos- del estigma y la discriminación asociados con la TB $(24,25)$.

El estereotipo del paciente con TBP consta de aspectos clínicos y sociales que, reunidos en un individuo, pueden favorecer la sospecha diagnóstica. Entre los elementos clínicos se destacan la infección por el virus de la inmunodeficiencia humana (VIH) y algunos signos, como la hemoptisis y la caquexia, característicos de estadios avanzados de la enfermedad. Entre los elementos sociales de ese estereotipo se encuentran la pobreza, el estar sin hogar, el alcoholismo, la drogadicción, la mala higiene y formar parte de una minoría étnica. Pero el estereotipo también puede entorpecer la oportunidad diagnóstica cuando las características del paciente no se ajustan a lo conocido, especialmente si los pacien- tes, el PS y la población general tienen conocimientos erróneos sobre el cuadro clínico y los factores de riesgo de esta enfermedad. Si bien la TBP se relaciona directamente con las categorías que configuran el estereotipo, se sabe que en zonas de alta prevalencia, la enfermedad no es exclusiva de poblaciones menos favorecidas ni de minorías étnicas. Esta categoría, emergente en este estudio, no había sido descrita como factor de retraso del diagnóstico en otros trabajos.

Por consiguiente, la falta de conocimientos y las creencias erróneas sobre la TBP y los enfermos contribuyen a fortalecer tanto el estigma como los estereotipos que lo soportan. En esta investigación se encontró que la TBP se percibía como un padecimiento grave, mortal, incurable, altamente contagioso e, incluso, como una enfermedad del pasado ya erradicada. Esta caracterización genera temor, no solo a la enfermedad sino a las personas que la padecen. Este temor hacia la TBP y los enfermos propicia la toma de medidas erróneas de aislamiento, algunas veces por recomendación del propio PS. De esta forma, las prácticas erróneas refuerzan no solo los estereotipos mencionados, sino también las actitudes de rechazo percibidas por los pacientes. Algunos autores han señalado que una de las causas de estigmatización y discriminación por enfermedades transmisibles, como la TBP, la lepra y el VIH/sida, es el excesivo hincapié en los cuidados y las manifestaciones exageradas de temor al contagio $(9,24)$.

Entre las conductas que pueden retrasar el diagnóstico se identificó el tratamiento sintomático sin prescripción médica al que acudieron frecuentemente los pacientes estudiados, lo que coincide con estudios realizados en otros países (26, 27). El PS debe entender que los pacientes tienen su propia visión de la enfermedad y deben acercarse a sus significados y saberes, pues es frecuente que las personas afectadas por una enfermedad combinen diferentes modelos del proceso salud-enfermedad (19).

$\mathrm{Al}$ igual que se ha observado en otros estudios $(28,29)$, el insuficiente conocimiento mostrado por el PS acerca de los factores de riesgo, la presentación clínica y el proceso de diagnóstico de la enfermedad, unido a la baja prescripción de la prueba de baciloscopia, redujeron la oportunidad diagnóstica en el grupo estudiado aquí. Por tratarse de una enfermedad endémica y de alta prevalencia
(30), esta situación es de gran importancia en la población estudiada, por lo que el PS debe contar con conocimientos básicos y adoptar un papel más activo en la detección temprana de la enfermedad.

En este estudio se encontró que el mayor tiempo de retraso en el diagnóstico de la TBP ocurrió después de que el paciente había consultado a un servicio de salud, algo ya informado por la OMS en 2006 (6) y constatado en estudios previos realizados en Colombia $(2,10)$.

Una limitación de este trabajo es no haber explorado la percepción del PS acerca del proceso de diagnóstico. Además, las categorías emergentes identificadas en esta investigación se basaron principalmente en las percepciones de los pacientes, por lo que es posible que algunos de ellos hayan tenido experiencias negativas que pudieran generar percepciones sesgadas. Sin embargo, en el taller realizado se validaron los resultados y hubo consenso al respecto. Por último, el retraso diagnóstico puede deberse a fallas en la estructura y el funcionamiento del modelo de salud (2), algo en lo que no se profundizó en este estudio.

A pesar de esas limitaciones, los resultados obtenidos indican que el PS desempeñó un papel fundamental en el diagnóstico tardío de la TBP en adultos de la ciudad de Medellín. Las creencias asociadas con el estigma social y el insuficiente conocimiento sobre la enfermedad - compartidas por el PS y los pacientes - contribuyen a la construcción social del estereotipo de la enfermedad y el enfermo. Esto puede hacer que no se piense en la TBP como posible diagnóstico cuando las características socioeconómicas y clínicas de los pacientes no se ajustan a ese estereotipo. Los conocimientos básicos del PS sobre la enfermedad eran inadecuados e insuficientes para ejercer una buena práctica diagnóstica, y estos se relacionaban con la baja sospecha de la enfermedad y el consecuente tratamiento sintomático, que retrasan la búsqueda de atención sanitaria por parte del paciente.

Para lograr un efecto positivo en la oportunidad del diagnóstico de la TBP se requiere mejorar la formación del PS - tanto en aspectos técnicos sobre la enfermedad como éticos relacionados con la práctica clínica- y se debe ofrecer mayor información sobre la TBP a la población general a fin de promover su detección temprana y romper con los estereotipos y el estigma social del enfermo y la enfer- 
medad. Se debe reconocer que la TBP es una enfermedad que puede afectar a todos y que es inaceptable que siga siendo causa de discriminación y aislamiento social.
Agradecimientos. La presente investigación recibió financiamiento de la Universidad de Antioquia, el Comité para el Desarrollo de la Investigación y la línea "Medellín social e incluyente" de la con- vocatoria temática Plan de Desarrollo (código CINV de la FNSP: INV222-06), apoyado por la Secretaría de Salud de Medellín.

\section{REFERENCIAS}

1. Colciencias. Contrato RC431/2004. Tuberculosis: la investigación integrada a la salud pública para mejorar su control. CCITB. Proyecto 1419. Medellín: Universidad de Antioquia; 2004.

2. Arbeláez MP, Gaviria MB, Franco A, Restrepo R, Hincapié D, Blas E. Tuberculosis control and managed competition in Colombia. Int J Health Plann Manage. 2004;19:25-43.

3. República de Colombia, Ministerio de la Protección Social. Situación de salud de Colombia. Indicadores básicos 2007. Santa Fe de Bogotá: Ministerio de la Protección Social; 2007. Hallado en http:/ / www.minproteccion social.gov.co/VBeContent/NewsDetail.asp? ID $=15895 \&$ IDCompany=3. Acceso el 31 de septiembre de 2009.

4. República de Colombia. Indicadores básicos de salud 2007. Departamento de Antioquia. Antioquia: Dirección Seccional de Salud y Protección Social de Antioquia; 2007. Hallado en http://www.dssa.gov.co/htm/indicadores. html. Acceso el 31 de septiembre de 2009.

5. World Health Organization. Global tuberculosis control: surveillance, planning, financing. WHO report 2005. Geneva: WHO; 2005. (WHO/HTM/TB/2005.349.) Hallado en http://www.who.int/tb/publications/global report/2005/en/index.html. Acceso el 30 de septiembre de 2009.

6. Coalición Antituberculosa para la Asistencia Técnica. Normas internacionales para la asistencia antituberculosa. La Haya: Coalición Antituberculosa para la Asistencia Técnica; 2006. Hallado en http:/ / www.theunion.org/ component/option,com_guide/id_down load, $92 /$ id_guide, 51 /task,OpenDown load/what,Guide\%20Complet\%20Esp/. Acceso el 15 de enero de 2010.

7. Pirkis JE, Speed BR, Young AP, Dunt DR, MacIntyre CR, Plant AJ. Time to initiation of anti-tuberculosis treatment. Tuber Lung Dis. 1996;77:401-6.

8. World Health Organization. Diagnostic and treatment delay in tuberculosis. An in-depth analysis of the health-seeking behaviour of patients and health system response in seven countries of the Eastern Mediterranean Region. Cairo: WHO; 2006. (WHO-EM/TDR/ 009/E/10.06/1000.) Hallado en http://www. emro.who.int/dsaf/dsa710.pdf. Acceso el 30 de septiembre de 2009.

9. Álvarez-Gordillo GC, Álvarez-Gordillo JF, Dorantes-Jiménez JE, Halperin-Frisch D. Percepciones y prácticas relacionadas con la tu42(6):520-8. berculosis y la adherencia al tratamiento en Chiapas, México. Salud Publica Mex. 2000;

10. Cáceres FM, Orozco LC. Demora en el diagnóstico de tuberculosis pulmonar en una región de Colombia. Rev Salud Publica. 2008;10(1):94-104.

11. Yimer $S$, Bjone $G$, Alene G. Diagnostic and treatment delay among pulmonary tuberculosis patients in Ethiopia: a cross sectional study. BMC Infect Dis. 2005;5:112.

12. Organización Panamericana de la Salud. Enseñanza de la tuberculosis en las facultades de salud. Informe de una consulta de expertos. Cartagena, Colombia, 6-8 de octubre de 2004. Washington, D.C.: OPS; 2005. (OPS/ DPC/CD/328-05.) Hallado en: http://www. paho.org/Spanish/AD/DPC/CD/tbfac-salud.pdf. Acceso el 23 de junio de 2009.

13. Stake RE. Investigación con estudios de casos. 3. ${ }^{a}$ ed. Madrid: Morata; 2005.

14. Yin R. Case study research: design and methods. 4th ed. Thousand Oaks, CA: Sage Publications; 2009. (Applied Social Research Methods Series Vol. 5.)

15. Creswell JW. Qualitative inquiry and research design. Choosing among five traditions. 2nd ed. Thousand Oaks, CA: Sage Publications; 2007.

16. República de Colombia, Ministerio de Salud. Resolución 412 del 25 de febrero de 2000. Santa Fe de Bogotá: Ministerio de Salud; 2000.

17. Geertz C. La interpretación de las culturas. 1 . $^{\mathrm{a}}$ ed. Barcelona: Gedisa; 2007.

18. Valencia S, Moscovici S. Representaciones sociales: alteridad, epistemología y movimientos sociales. Guadalajara: Universidad de Guadalajara; 2006.

19. Candreva A, Paladino C. Cuidado de la salud: el anclaje social de su construcción, estudio cualitativo. Univ Psychol. 2005;4(1): $55-62$.

20. Jaramillo E. The impact of media-based health education on tuberculosis diagnosis in Cali, Colombia. Health Policy Plan. 2001;16 (1):68-73.

21. Corona AE, Morales G, Chalgub AM, Armas L, Acosta OS, González E. Conocimientos, percepciones y prácticas de grupos de población respecto a la tuberculosis, 19941996. Rev Cubana Med Trop. 2000;52(2): $110-4$.

22. Cambanis A, Yassin MA, Ramsay A, Squire SB, Arbide I, Cuevas LE. Rural poverty and delayed presentation to tuberculosis services in Ethiopia. Trop Med Int Health. 2005;10: 330-5.

23. Goffman E. Estigma. La identidad deteriorada. Buenos Aires: Amorrurtu Editores; 2003.

24. Baral S, Karki D, Newell J. Causes of stigma and discrimination associated with tuberculosis in Nepal: a qualitative study. BMC Public Health. 2007;7(211):1-10.

25. Bassili A, Seita A, Baghdadi S, Alabsi A, Abdilai I, Agboatwalla M, et al. Diagnostic and treatment delay in tuberculosis in 7 countries of the Eastern Mediterranean region. Infect Dis Clin Pract. 2008;16(1):23-35.

26. Altet MN, Alcaide J, Canela J, Milá C, Jiménez MA, De Souza ML, et al. Estudio del retraso diagnóstico de la tuberculosis pulmonar sintomática. Arch Bronconeumol. 2003;39: $146-52$.

27. Muñoz D, Ríos G, Villalva C, Muñoz S. Factores asociados al diagnóstico tardío de pacientes con tuberculosis pulmonar en Lima Este, Perú. Rev Peru Med Exp Salud Publica. 2004:21(1):18-22.

28. República de Nicaragua, Ministerio de Salud. Conocimientos, actitudes y prácticas que tienen los pacientes, familiares, personal de salud y población en general sobre la tuberculosis, con enfoque antropológico en $36 \mathrm{mu}-$ nicipios de siete SILAIS de Nicaragua, 2004. Informe final. Managua: Ministerio de Salud; 2004. Hallado en http://www.mcp.org.ni/ proyecto/tuberculosis/media/CAP.en.TB.p df. Acceso el 30 de septiembre de 2009.

29. Uplekar M, Pathania V, Raviglione M. Private practitioners and public health: weak links in tuberculosis control. Lancet. 2001;358(9285): 912-6.

30. Giraldo MR, Álvarez HB, Gutiérrez V. Día Mundial de la Tuberculosis 2007. De la acción local a la eliminación global: TB aquí es TB en todas partes. Boletín Información para la Acción-BIA. Medellín: Dirección Seccional de Salud Pública de Antioquia; 2007. Hallado en http:/ / www.dssa.gov.co/dowload/BIA032007.pdf. Acceso el 23 de junio de 2009.

Manuscrito recibido el 22 de enero de 2009. Aceptado para publicación, tras revisión, el 24 de junio de 2009 
ABSTRACT Objective. To describe the role of health care providers in the delayed diagnosis of pulmonary tuberculosis (PTB) among the adult population in a city in Colombia.

\section{The role of health care providers in the late diagnosis of pulmonary tuberculosis among adults in Medellín, Colombia}

Methods. A case study of PTB diagnosis among adults in the city of Medellín, Colombia, in 2007. Quantitative and qualitative data were collected through in-depth interviews with 19 patients (11 with early diagnosis and 8 with late diagnosis), from medical records, and by a survey of PTB-related knowledge and practices among 89 doctors and nurses at health care clinics. A research workshop was held with patients to share the results and validate new categories.

Results. A lack of PTB knowledge and patient stereotyping were found to lead to misinterpretation of symptoms and the use of symptomatic treatments. The lack of knowledge regarding risk factors and clinical protocols made diagnosis difficult when the patient did not match the stereotype or presented with a more well-known illness. Misinformation on PTB transmission and inappropriate measures led to discriminatory practices and social isolation and only reinforced stereotypes and stigma, which in turn discouraged the quest for medical attention.

Conclusions. Health care providers played a large part in the late diagnosis of PTB in the adults studied. Beliefs regarding social stigma and a lack of knowledge about the disease - shared by providers and patients-contributed to stereotyping of the illness and the disease-carrier, and limited the probability of an early diagnosis of PTB.

Key words Tuberculosis, pulmonary; delayed diagnosis; health personnel; stereotyping; Colombia. 